\title{
Nanomedicine Strategies to Enhance Tumor Drug Penetration in Pancreatic Cancer
}

Tao Lu

Jai Prakash

Engineered Therapeutics Group, Department of Biomaterials Science and Technology, University of Twente, Enschede, The Netherlands
Correspondence: Jai Prakash Engineered Therapeutics, Department of Biomaterials Science and Technology, University of Twente, Drienerlolaan 5,

Enschede, 7500AE, The Netherlands

Tel +3I 534893096

Email j.prakash@utwente.nl

\begin{abstract}
Pancreatic cancer is one of the most malignant tumors with one of the worst survival rates due to its insidious onset and resistance to therapies. Most therapeutics show a desired anticancer effect in vitro; however, very poor efficacy in vivo because of the limited drug delivery and penetration into pancreatic tumors attributed to the abundance of the tumor stroma, ie, the fibrotic tumor microenvironment surrounding the cancer cells. For a better understanding of the challenges posed by the pancreatic tumor stroma, we outline the key features of the tumor microenvironment. Then we highlight major strategies used to tackle the challenges to improve drug penetration into the tumor and achieve enhanced efficacy (pre)clinically. Furthermore, we describe nanomedicine strategies to modulate the tumor stroma, degrade the extracellular matrix, and co-deliver multi-functional drugs, to improve the chemotherapeutics delivery and penetration into pancreatic tumors.
\end{abstract}

Keywords: pancreatic ductal adenocarcinoma, cancer-associated fibroblasts, tumor stroma, tumor vasculature, drug perfusion, drug penetration

\section{Introduction}

Pancreatic cancer is one of the most fatal solid tumors in the world, with a very low 5year overall survival rate of less than $8 \% .{ }^{1}$ Based on the GLOBOCAN estimation in 2020, pancreatic cancer is listed the seventh leading cause of death worldwide in both males and females, with the highest incidence rates in Europe and Northern America. ${ }^{2}$ Pancreatic cancers are generally classified as endocrine cancer and exocrine cancer, among which pancreatic ductal adenocarcinoma (PDAC) is an exocrine tumor and accounts for around $90 \%$ of all malignant pancreatic neoplasms. ${ }^{3}$ Due to its poor diagnosis and prognosis, the number of deaths resulting from PDAC almost equalizes with the number of cases. ${ }^{2}$ According to a report, 458,918 PDAC cases were diagnosed, with 432,242 deaths worldwide in 2018. ${ }^{4}$ A study from 28 European Union countries predicts that pancreatic cancer may become the third leading cause of cancer-related deaths in Europe by $2025 .^{5}$ Nowadays surgical treatment, chemotherapy, and radiotherapy are still the routine methods in the clinic. However, surgical resection is reported to be applicable in only $10-15 \%$ of patients with PDAC, as most PDAC cases, due to the nonspecific clinical symptoms, are diagnosed at the advanced stage which usually presents widespread metastasis already. ${ }^{6,7}$ Moreover, development of high resistance has been observed in PDAC against traditional chemotherapy and radiotherapy, attributing to the complex tumor microenvironment and aggressive nature of PDAC, leading to most treatments being ineffective. ${ }^{8}$ Currently, gemcitabine is used as alone or in combination with other cytotoxic agents, 
and is applied as the first-line chemotherapy for PDAC in the clinic. ${ }^{8}$ However, mostly only a few months of survival extension after treatment are observed for patients due to the accumulated side-effects resulting from systemic administration, non-specific drug target, and limited drug delivery in tumors. ${ }^{9-12}$

A typical feature of PDAC is the abundant desmoplasia, ie, highly fibrotic stroma in the tumor which can occupy up to $90 \%$ of the total tumor mass. ${ }^{13}$ The interplay between the tumor microenvironment and cancer cells promotes this desmoplastic reaction, leading to formation of a dense stroma in PDAC. Several studies have shown that a higher stromal content is associated with poor survival rate. ${ }^{14}$ The PDAC stroma closely surrounds the cancer cells and interacts with these cells in both a paracrine and juxtacrine manner. The tumor stroma is composed of extracellular matrix (ECM) and various stroma cells, such as cancerassociated fibroblasts (CAFs) or pancreatic stellate cells, inflammatory immune cells such as tumor-associated macrophages (TAMs), natural killer (NK) cells, and vascular-cells including endothelial cells and pericytes ${ }^{15}$ (Figure 1). In addition, the distorted blood vessels promotes hypoxia in the tumor, which drives the chemoresistance. ${ }^{16}$

\section{Nanomedicine to Treat Pancreatic Cancer}

The development of nanotechnology to formulate a nanoscale drug delivery system provides possibilities to achieve improved therapy outcome, such as prolonged circulation, reduced side-effects, and site-specific drug delivery. ${ }^{17,18}$ Nanocarriers can enter tumors via the phenomenon socalled Enhanced Permeability and Retention (EPR) effect - the process which occurs due to the immature and leaky blood vessels allowing nanocarriers to enter the tumor and poor lymphatic system which does not allow them to escape. ${ }^{19}$ However, the EPR effect varies in patients depending on the type of tumor and vascularization. ${ }^{20}$ In addition, due to the inherent property of nanocarrier-based delivery systems, they are able to load a high amount of chemotherapeutics which allow them to deliver a higher drug concentration to the tumor site in vivo compared to free drug. In 2013, albumin-bound paclitaxel nanoparticle

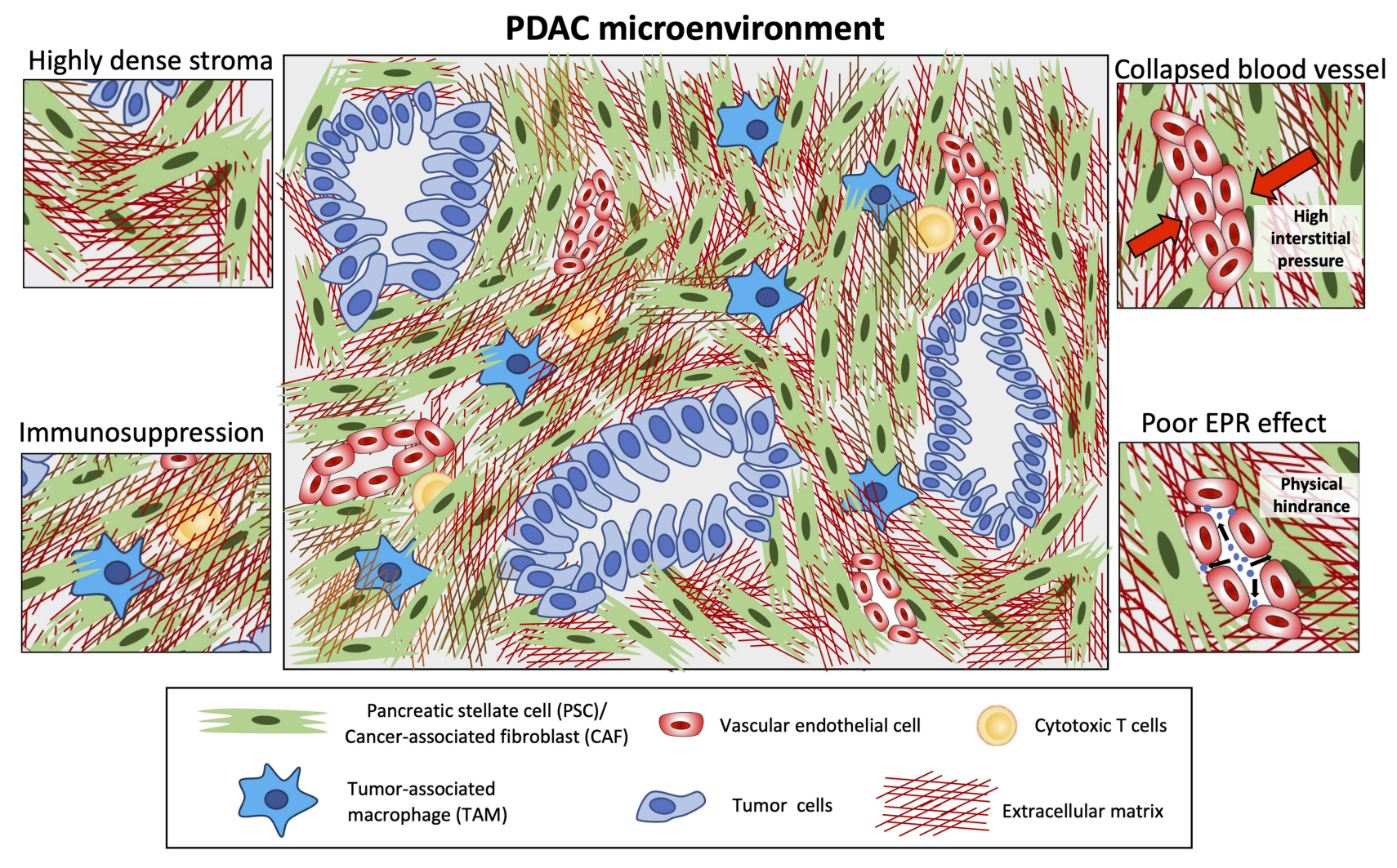

Figure I Key features of the PDAC microenvironment. This diagram highlights highly dense stroma and its impact on immune infiltration (entrapped immune cells) leading to immunosuppression, collapsed blood vessels due to high interstitial pressure, and poor enhanced permeability and retention (EPR) effect hindering nanoparticles to penetrate into the tumor. 
Table I Pancreatic Cancer Clinical Trials of Nanomedicine Strategies for Drug Delivery (Referring from https://clinicaltrials.gov/)

\begin{tabular}{|l|l|}
\hline Agent and Nanotechnology Approach & $\begin{array}{l}\text { Status and Relevant } \\
\text { Findings }\end{array}$ \\
\hline NASOX: liposomal irinotecan with S-I and oxaliplatin & Phase I/lla \\
PanDox: thermoliposome doxorubicin & Phase I \\
Liposomal irinotecan with vactosertib, 5-FU and LV & Phase I \\
AGulX gadolinium-chelated polysiloxane based nanoparticles with radiotherapy & Phase I/II \\
NBTXR3: hafnium oxide-containing nanoparticles with radiotherapy & Phase I \\
Nanoparticle paclitaxel albumin-bound protein in combination with ascorbic acid, cisplatin and gemcitabine & Phase I/II \\
Nanoparticle paclitaxel albumin-bound protein in combination with 5-FU, gemcitabine, cisplatin, irinotecan and folinic & Phase II \\
acid and radiotherapy & \\
\hline
\end{tabular}

(nab-paclitaxel) was approved to use in combination with gemcitabine as the frontline treatment for advanced stage pancreatic cancer patients. ${ }^{21}$ Paclitaxel was proposed to reduce the tumor stroma content and the expression of gemcitabine-metabolizing enzyme in patients with PDAC, thus improving the efficacy of gemcitabine in the tumor and leading to a significant survival rate. ${ }^{17}$ Although an effect on tumor stroma was seen, this is likely non-specific and due to a general cytotoxic effect on CAFs, which resulted in the stroma depletion. Nevertheless, nab-paclitaxel has delivered improved therapeutic efficacy in the clinic. In 2015, another nanomedicine, MM-398, a liposomal formulation encapsulating irinotecan, the topoisomerase I inhibitor was approved as a combinational therapy with 5-fluorouracil and folinic acid, for the second-line treatment of metastatic PDAC patients. ${ }^{17}$ This combination therapy showed an improved overall survival in patients compared to 5-fluorouracil and folinic acid therapy alone due to the enhanced intratumoral drug level of irinotecan. ${ }^{22,23}$ Currently, growing nanomedicines for PDAC treatment have been undergoing clinical trials, which are summarized in Table 1.

\section{Tumor Penetration Challenges in PDAC}

Despite having some successful examples of nanomedicine against PDAC, nanomedicine still faces great challenges for its penetration into the tumor. The accumulation of nanomedicine into tumors depends on the physical microenvironment of the tumor, including tumor vascularization (abundance of blood vessels and maturity of vessels), interstitial fluid pressure, tumor stroma content, and tumor lymphatic flow. Unfortunately, the PDAC microenvironment represents one of the most unfavorable features for the penetration of nanomedicine. PDAC is a poorly vascularized tumor type with dense tumor stroma in the surrounding, with tightly packed CAFs within the ECM. CAFs produce enormous amounts of ECM such as collagen and promote their crosslinking by producing lysyl oxidase (LOX) enzymes. Furthermore, due to the contractile nature, CAFs contract the ECM and enhance tumor stiffness (rigidity and elasticity) within PDAC. Wang et $\mathrm{al}^{24}$ have demonstrated that tissue stiffness measured using elastography correlates with drug delivery in orthotopic PDAC models. They proposed that shear modulus (a measurement of stiffness) increases with increased collagen density and is inversely related to drug delivery. Furthermore, Nia et $\mathrm{al}^{25}$ suggested that drug delivery is not only dependent on the tissue stiffness but also on the solid stress which is caused by increased cell proliferation, matrix deposition, and gel swelling. Since the lymphatic vessels in PDAC are non-functional, the accumulated fluid is not able to escape. Besides that, ECM molecules such as hyaluronic acid, that has a high capability of retaining fluids, swell and increase the tumor interstitial fluid pressure (IFP). High IFP leads to the compression of tumor blood vessels and sometimes a full collapse, impairing the perfusion of small molecules to nanomedicine into the tumor. Furthermore, the ECM proteins such as fibrillar collagens form a complex network which acts as a physical barrier for the penetration of macromolecules, nanomedicine, as well as immune cells. Consequently, due to collapsed blood vessels and a high stroma barrier the EPR effect is minimal in PDAC.

In summary, the main features of PDAC turning them into highly impenetrable to small drug molecules, nanocarriers and immunotherapies are illustrated in Figure 1 and summarized in Table 2. Developing new strategies is urgently needed to improve the bioavailability of drugs to the tumor. Below we discuss the current progress on different strategies to increase the intratumoral drug uptake in pancreatic cancer. 
Table 2 Summarizing the PDAC Features Effecting Tumor Perfusion and Penetration and Potential Solutions

\begin{tabular}{|c|c|c|c|}
\hline Key Features of PDAC & Mechanisms & Impact on Different Therapeutics & $\begin{array}{l}\text { Potential Strategies to Enhance } \\
\text { Penetration }\end{array}$ \\
\hline $\begin{array}{l}\text { Highly dense stroma } \\
\text { (Increased stiffness and } \\
\text { solid stress) }\end{array}$ & $\begin{array}{l}\text { - Excessive ECM } \\
\text { production by CAFs } \\
\text { - Reduced ECM } \\
\text { degradation } \\
\text { - Crosslinked matrix } \\
\text { - Contractile CAFs }\end{array}$ & $\begin{array}{l}\text { Poor diffusion and penetration of } \\
\text { - Macromolecules, eg, monoclonal antibodies } \\
\text { - Nanomedicine }\end{array}$ & $\begin{array}{l}\text { - Inducing ECM degradation } \\
\text { - Targeting CAFs (inhibit CAF- } \\
\text { induced matrix production and CAF } \\
\text { contraction) }\end{array}$ \\
\hline $\begin{array}{l}\text { Compressed and } \\
\text { collapsed vasculature }\end{array}$ & $\begin{array}{l}\text { - Infantile } \\
\text { vasculature } \\
\text { - High interstitial } \\
\text { fluid pressure } \\
\text { - Non-functional } \\
\text { lymphatics }\end{array}$ & $\begin{array}{l}\text { Poor perfusion of } \\
\text { - Small drug molecules, eg, chemotherapeutics } \\
\text { - Nanomedicine }\end{array}$ & $\begin{array}{l}\text { - Reduce IFP using hypotensive agents } \\
\text { - vasculature normalization }\end{array}$ \\
\hline Poor EPR* effect & $\begin{array}{l}\text { - Compressed and } \\
\text { collapsed blood } \\
\text { vessels } \\
\text { - Excessive matrix } \\
\text { deposition }\end{array}$ & $\begin{array}{l}\text { Reduced extravasation } \\
\text { - Macromolecules, eg, monoclonal antibodies } \\
\text { - Nanomedicine }\end{array}$ & $\begin{array}{l}\text { - Improve blood perfusion, eg, } \\
\text { normalization of vasculature } \\
\text { - Enhance ECM degradation and } \\
\text { inhibit ECM deposition }\end{array}$ \\
\hline $\begin{array}{l}\text { Immuno-suppressive } \\
\text { environment }\end{array}$ & $\begin{array}{l}\text { - Interaction } \\
\text { between TAMs and } \\
\text { cancer cells } \\
\text { - Dense stroma }\end{array}$ & $\begin{array}{l}\text { - Poor immuno-surveillance and efficacy of } \\
\text { immunotherapy, eg, cell-based therapies and } \\
\text { checkpoint inhibitors }\end{array}$ & $\begin{array}{l}\text { - Targeting immune cells, eg, } \\
\text { reprogram TAMs into anti-tumoral } \\
\text { type } \\
\text { - Anti-stromal agents to enhance } \\
\text { penetration }\end{array}$ \\
\hline
\end{tabular}

Abbreviation: *EPR, Enhanced Permeability and Retention effect.

\section{Strategies to Improve Tumor Penetration}

To improve the penetration of (nano)drug into PDAC, many strategies have been proposed which are summarized in Figure 2. These strategies can be divided into two major categories: 1) Modulating nanomedicine physicochemical properties; and 2) Modulation of tumor stroma.

\section{Modulation of Nanomedicine Size Reducing Strategies to Improve Penetration}

Reducing the size of nanoparticles may facilitate the penetration into tumor. Li et al $^{26}$ developed a size-switchable nanoparticle with cisplatin loaded to treat pancreatic tumor. This nano-system, with an initial volume around $80 \mathrm{~nm}$, was triggered by the acidic environment in the tumor tissues ( $\mathrm{pH}$ 6.5-7.0) and then dissociated into $\sim 10 \mathrm{~nm}$ sized dendrimer building blocks, showing significantly deeper intratumoral penetration and thus improved efficacy. ${ }^{26}$ Cun et $\mathrm{al}^{27}$ designed a dual-loading nano-system that was composed of a large nanoparticle with $18 \beta$-glycyrrhetinic acid loaded which regulated CAFs, and small nanoparticles with gemcitabine encapsulation which exhibited deeper penetration after releasing from the big particles in response to MMP-2 in the pancreatic tumor model. Li et al ${ }^{28,29}$ reported a series of poly(amidoamine)-based small nanoparticles which achieved a deep tumor penetration. They developed a light sensitive, oxygen-responsive copolymeric vesicle system containing hydrogen peroxide and poly(amidoamine) dendrimer conjugating chlorin e6/cypate (CC-PAMAM) to generate oxygen and release the small photoactive CC-PAMAM particles for the deep penetration in tumor, which showed a significant inhibition of tumor growth in a human pancreatic tumor model. ${ }^{30} \mathrm{~A}$ recent study from Sun et $\mathrm{al}^{31}$ applied a gemcitabine-conjugated polymer to co-load with paclitaxel and an immunomodulating agent in a micelle system with a diameter of $\sim 15 \mathrm{~nm}$. They showed that this small nanoparticle induced clearly increased drug accumulation compared to large-sized nanoparticles $(\sim 160 \mathrm{~nm})$, thus improved efficacy in the PDAC tumor model. ${ }^{31}$ Taken together, nanoparticles with reduced size may lead to an improved intratumoral 


\section{Engineering nanomedicine}

\section{Size reduction strategy $\rightarrow$ enhanced penetration of small nanoparticles}
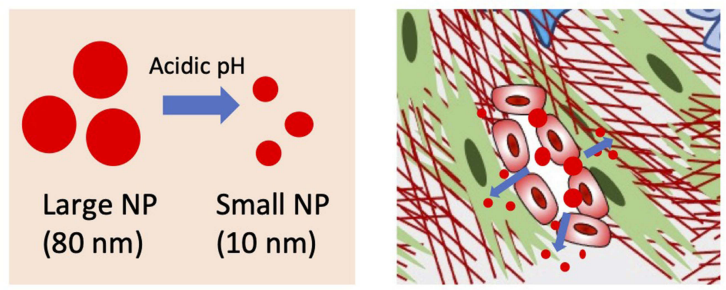

Triggered burst release $\rightarrow$ enhanced diffusion of small molecules
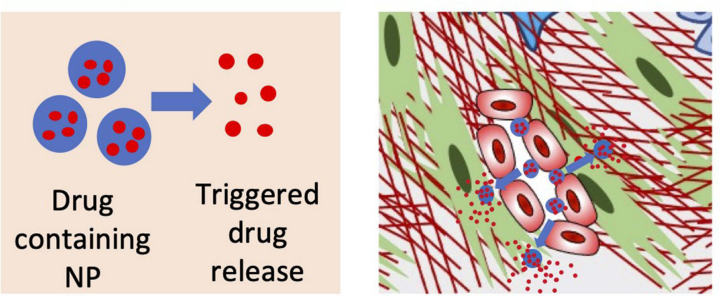

Transcytosis $\rightarrow$ enhanced penetration through cells
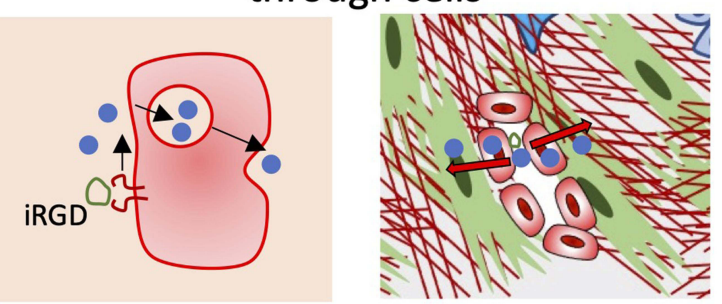

\section{Reprogramming tumor stroma}
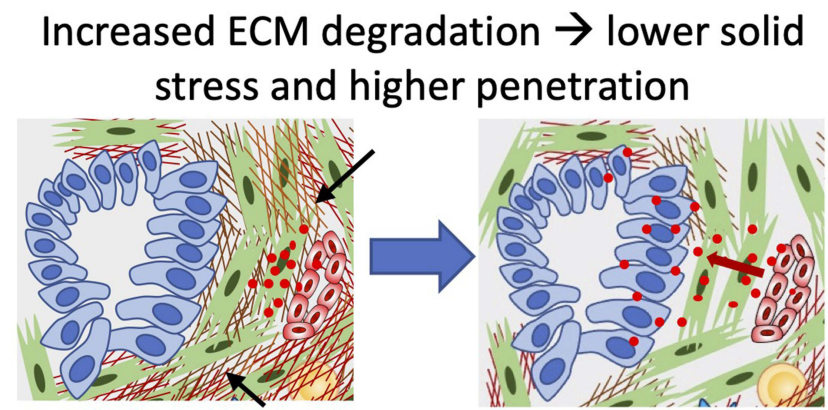

Normalization of CAFs $\rightarrow$ reduced stiffness and better penetration
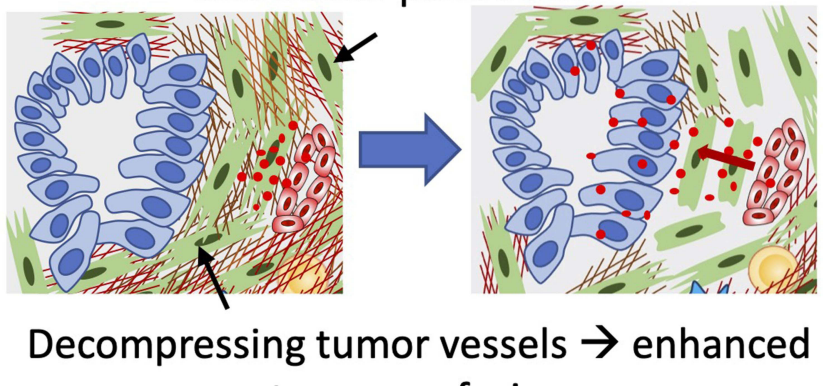
tumor perfusion

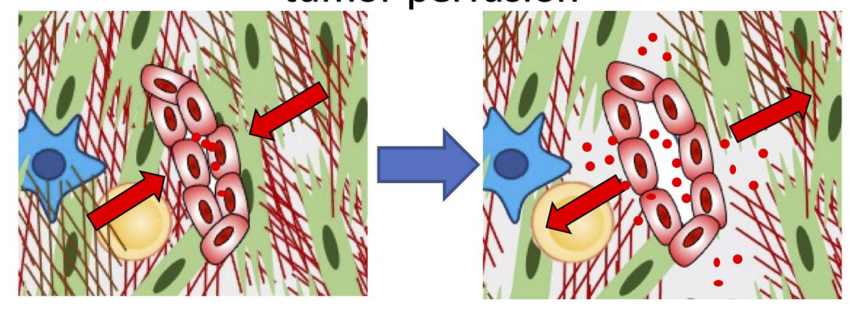

Figure 2 Diagrammatic representation of different strategies to enhance tumor penetration of nanomedicine. To enhance the penetration, either nanomedicine can be engineered or the tumor stroma can be re-programmed. Nanomedicines can be tuned by size reduction strategy in which the size of nanoparticles (NP) is reduced upon reaching the tumor site, which allows deeper penetration into the tumor. In another strategy, one may induce triggered release of drug allowing rapid diffusion of small molecules into the tumor. In the third strategy, use of a transcytosis mechanism is interesting to induce penetration of nanoparticles through endothelial cells, for example using iRGD peptide. Other approaches are based on reprogramming of tumor stroma. In this approach, one may induce degradation of ECM using enzymes such as MMPs to reduce the physical barrier and solid stress or by reducing stiffness by normalization of CAFs. These approaches help in enhancing penetration by modulating physical microenvironment. Although reduction of ECM will lead to decompression of blood vasculature, relaxation of blood vessels using anti-hypertensive agents can induce tumor blood perfusion.

distribution and thus achieve an improved therapeutic efficacy. While this strategy is not suitable for different types of payloads due to their inability to be encapsulated in dendrimers, the concept can be explored to develop new systems based on other nanomaterials.

\section{Triggered Local Release to Enhance Drug Uptake}

Locally quick-trigger release of drug in the tumor may also offer an alternative strategy to increase chemotherapeutics uptake by tumor tissues, due to the fact that as the result of rapid intravascular release, the established high drug concentration gradient between tumor vessel and tumor interstitium drives these small drug molecules diffusion and promotes penetration and uptake in the tumor. $\mathrm{Lu}$ et al and others have shown the advantages of using these quick-triggered local drug release nanosystems to improved drug uptake by different tumor models. ${ }^{32-35}$ Similarly, Malekigorji et $\mathrm{al}^{36}$ reported an externally thermally-triggered nano-drug delivery system in pancreatic tumor models. They observed a clear tumor growth inhibition after applying a local irradiation which triggered the release of drug from the nanoparticles, improving tumor take-up of the drugs. ${ }^{36}$ Emamzadeh et al $^{37}$ synthesized a 
novel thermoresponsive copolymer (poly(diEGMA-coOEGA300)-b-PEHMA) to self-assemble into micelles which showed a heat-triggered drug release at mild hyperthermia of $40^{\circ} \mathrm{C}$. They observed an increased cytotoxicity after applying hyperthermia in a pancreatic cancer in vitro study. ${ }^{37}$ These studies suggest that quick-trigger release nano-systems may offer an alternative manner to improve drug penetration and uptake in pancreatic tumor treatment.

\section{Transcytosis Using Specific Peptides}

Lo et $\mathrm{al}^{38}$ designed tumor-penetrating nanocomplexes by utilizing cyclic iRGD peptide (targeting $\alpha_{\mathrm{v}} \beta_{3 / 5}$ integrins and neuropilin-1) with a cell penetrating peptide to deliver anti-Kras siRNA to PDAC. They showed that the nanocomplexes delivered anti-Kras siRNA into a PDAC mouse model and significantly delayed the tumor growth. In order to enhance the penetration across the tumor, a transcytosis mechanism has been explored. Transcytosis refers to the transfer of molecules from one side of the cell to another side that involves endocytosis, transfer of vesicle, and exocytosis. Wang et $\mathrm{al}^{39}$ developed a polyamidoamine dendrimer-camptothecin conjugate that actively penetrates deep into PDAC tissue through $\gamma$-glutamyl transpeptidase-triggered cell endocytosis and transcytosis. In vivo, this dendrimer-drug conjugate exhibited high antitumor activity in patient-derived xenograft and orthotopic tumor models compared to gemcitabine for advanced pancreatic cancer. A study from Chen et $\mathrm{al}^{40}$ demonstrated a central stroma targeting strategy to improve drug delivery in pancreatic tumors. They developed peptide conjugated micelles with $\mathrm{pH}$-sensitivity to specifically target the UPAR (urokinase type plasminogen activator receptor), a receptor overexpressed in tumor and stroma cells, which showed more nanoparticle accumulation in the tumor and selectively released chemotherapeutics in the low $\mathrm{pH}$ part of the tumor (eg, center of stroma or lysosome), significantly improving the efficacy. The strategy based on transcytosis is highly interesting to efficiently deliver nanoparticles across endothelial cells and stromal cells. More experimental evidence is, however, required to establish the translational value of this concept in vivo in terms of tumor specificity, applicability in highly desmoplastic and heterogenic tumors, and off-target effects.

\section{Modulation of Tumor Stroma} Targeting CAFs to Improve Penetration

The PDAC microenvironment is enriched with a large number of stromal cells, in particular CAFs are the most abundant cell type. During tumorigenesis, stromal cells are either derived from resident cells or recruited from the bone marrow. Once recruited, they get activated by neoplastic cell-derived cytokines, chemokines, and exosomes, creating a tumor-favoring microenvironment, which further promotes the tumor development. ${ }^{41,42}$ In pancreatic cancer, pancreatic stellate cells (PSCs) are the most abundant cell type in the stroma, constituting around $50 \%$ of it. ${ }^{43}$ Within the tumor microenvironment, the quiescent PSCs can be stimulated into the activated form and then differentiated into different subtypes of CAFs such as myofibroblastic CAFs (myCAFs), inflammatory CAFs (iCAFs), and (ap-CAFs). ${ }^{44-46}$ Moreover, more subtypes might be present in the complex microenvironment. MyCAFs (defined by $\alpha-\mathrm{SMA}^{+}, \mathrm{FAP}^{+}$), mainly activated by transforming growth factor (TGF)- $\beta$, are largely responsible for the synthesis and production of ECM proteins such as collagen, fibronectin, epidermal growth factor (EGF), connective tissue growth factor (CTGF), and matrix metalloproteinases (MMPs). iCAFs $\left(\alpha-\mathrm{SMA}^{ \pm}\right.$, $\left.\mathrm{FAP}^{ \pm}, \mathrm{IL}^{+}\right)$are the cytokines-secreting phenotype which are crucial for cell-cell interaction via paracrine signal. Ap-CAFs $\left(\mathrm{CD}^{+} 4^{+}, \mathrm{MHCII}^{+}\right)$display immunomodulatory effects via activation of cytotoxic T-cells. Altogether, these features of different CAFs are essential to the pancreatic tumor growth, invasion, and resistance to chemotherapeutics. $^{43,47,48}$

Increasing studies have shown that the CAF targeting strategy may provide improved drug delivery into the tumor. There are a number of experimental studies which have shown the use of nanoparticles to either co-encapsulate an anti-stromal drug and chemotherapeutics into a nanoparticle or combine anti-stromal drug with a nanoparticle containing chemotherapeutic agent. Sonic hedgehog (SHH), secreted by tumor cells, is reported to stimulate PSCs to significantly promote the formation of desmoplasia in pancreatic tumors. ${ }^{49-51}$ Bailey et $\mathrm{al}^{50}$ observed a significant decrease of desmoplastic reaction in PDAC after using the antibody to inhibit SHH signaling. A landmark study from Olive et $\mathrm{a}^{52}$ reported the semi-synthesized IPI-926 which was expected to block the SHH pathway via inhibiting the hedgehog receptor Smoothened in CAFs to improve drug delivery, as 
Smoothened is highly upregulated in pancreatic CAFs. ${ }^{49}$ They found that IPI-926 increased the vascular density and reduced desmoplasia, and thus improved gemcitabine concentration in the tumor. ${ }^{52}$ However, in the follow-up clinical study IPI-926 was halted in a Phase II trial due to the increased mortality compared to the control group in pancreatic cancer treatment. ${ }^{53}$ One explanation could be from the work by Lee et al, ${ }^{54}$ who found that when Smoothened is inhibited in the SHH pathway, it accelerates the progression and metastasis of PDAC while indirectly decreasing desmoplasia. Besides, Rhim et $\mathrm{al}^{55}$ also observed a more aggressive tumor development after deleting the $\mathrm{SHH}$ in a PDAC mouse model. A recent clinical study of vismodegib, another SHH inhibitor, failed to show efficacy advantages in combination with chemotherapeutics in patients. ${ }^{56}$ Similarly, Ozdemir et $\mathrm{al}^{57}$ reported that depletion of CAFs in pancreatic tumors induced immunosuppression, promoted tumor growth, and reduced survival. Although these studies raised concerns about the potential tumorprogressing role of CAFs, it also triggered the better understanding of the heterogeneity of CAFs, pathwayrelated effects, and discrepancies due to the total depletion of CAFs. Considering these issues, modulation of CAFs, preferably specific subtypes, rather than removal of all CAFs might provide improved therapeutic benefits. ${ }^{58}$

Integrins are a cell adhesion receptors family consisting of $\alpha$ and $\beta$ subunits. Integrins are proposed as an important player in mediating cell migration, proliferation, survival, and signal transduction between the cell and ECM. $^{59,60}$ Previously we have shown that integrin $\alpha 11$ was overexpressed in activated fibroblasts of pancreatic tumors, making it a potential target for CAFs. ${ }^{61}$ After knockdown of integrin $\alpha 11$, an inhibition of activation of PSCs was observed, showing a reduction in differentiation, ECM production, and contraction. Furthermore, PSCs with $\alpha 11$ knockdown showed a reduced tumor cell migration enhancing effect compared to activated PSCs. ${ }^{61}$ Recently, another study from our group reported that the overexpression of integrin $\alpha 5$ in PDAC tumor stroma can be an interesting therapeutic target in CAFs. ${ }^{14}$ Interestingly, knockdown of $\alpha 5$ in PSCs led to the reduced activation of PSCs in vitro and reduced desmoplasia in vivo in mouse xenograft co-injection tumor models. Besides that, we demonstrated that using an antagonist against integrin $\alpha 5$ could reduce the desmoplasia (collagen content), resulting into decompression of blood vessels in co-injection and patient-derived xenograft mouse models. This decompression led to the enhanced tumor perfusion and therefore higher efficacy of gemcitabine into these mouse tumor models. ${ }^{14}$

Connective tissue growth factor (CTGF) is highly expressed in PDAC tissues including CAFs and, in turn, this factor also induces CAFs proliferation, migration, and ECM deposition. ${ }^{62,63}$ Aikawa et al $^{64}$ demonstrated that using CTGF-specific monoclonal antibody (FG-3019) significantly inhibited tumor growth, metastasis, and angiogenesis in a mouse pancreatic cancer model. The following clinical trials indicated that patients who were treated with FG-3019 had increased potential for surgery and survival. ${ }^{65}$ These promising results have also granted this regimen for the Phase III clinical trial recently. ${ }^{65} \mathrm{~A}$ study from Resov et al ${ }^{66}$ investigated two synthetic peptides targeting CTGF and showed an increased tumor inhibition when administered in combination with gemcitabine. It is worthy to note that the peptide did not improve the gemcitabine level in the PDAC tumor model. ${ }^{66}$

Furthermore, Han et $\mathrm{al}^{67}$ reported a dual-enzyme-sensitive nano-system with gemcitabine encapsulation to target pancreatic tumors, which showed significantly improved tumor inhibition via the targeted uptake and maintenance of gemcitabine in the tumor cells. A study from the same group demonstrated a strategy of using metformin to disrupt the dense stroma followed by gemcitabine-loaded nanoparticles treatment. ${ }^{68}$ Metformin, an antihyperglycemic drug, is reported to suppress tumor growth by activating the AMPK (adenosine monophosphate activated kinase) pathway which is closely associated with desmoplasia via downregulation of TGF- $\beta .^{69}$ The authors observed an increase of gemcitabine nanoparticle accumulation in the tumor after metformin therapy, which showed significant suppression of tumor growth compared with other groups. ${ }^{69}$

Pirfenidone, an antifibrotic drug, is commonly used to treat the idiopathic pulmonary fibrosis by down-regulating the TGF- $\beta$ signaling pathway and collagen synthesis. ${ }^{70,71}$ Kozono aet $\mathrm{al}^{72}$ reported that using pirfenidone to target the PSCs inhibited the proliferation, invasion, migration, and secretion of collagen, fibronectin, and periostin in the pancreatic cancer cell-activated PSCs. In addition, it showed a significant reduction of peritoneal dissemination and liver metastasis as well as the overall tumor growth in the PDAC mouse model when co-administrating pirfenidone and gemcitabine compared with gemcitabine alone. ${ }^{72}$ A recent study from Gao et $\mathrm{al}^{73}$ investigated a $\mathrm{pH}$-triggered nanosystem to co-deliver pirfenidone and gemcitabine to the pancreatic tumor, which showed reduced 
desmoplasia of tumor stroma, thus promoting drug penetration in the tumor and improved efficacy. $\mathrm{Ji}$ et $\mathrm{al}^{74}$ designed a MMP-2 responsive liposome with pirfenidone (PFD) encapsulated to down-regulate the components of ECM first, followed by gemcitabine treatment. The result showed a clearly deeper penetration of drug after the liposomal PFD treatment, thus leading to improved tumor inhibition. ${ }^{74}$

PSCs are known to store vitamin A (retinol)-storing lipid droplets inherently, which they lose when activated. ${ }^{75}$ As a consequence, PDAC patients are generally deficient in this fat-soluble vitamin, which further promotes the activation of PSCs. ${ }^{76,77}$ Evidence has shown that using all-trans retinoic acid (ATRA) can restrain the desmoplasia formation and inhibit the tumor growth in PDAC, making it a promising agent for activated PSC-targeted therapy. ${ }^{76,78,79}$ Han et $\mathrm{al}^{80}$ utilized a $\mathrm{pH}$-responsive nanosystem to deliver ATRA in a PDAC mouse model. This treatment induced quiescence of PSCs and inhibited the ECM accumulation significantly, thus providing an increased delivery of gemcitabine into the tumor. A recently finished Phase I trial of ATRA in combination of gemcitabine and nab-paclitaxel in pancreatic cancer treatment has supported the validity, showing expected stroma normalization after administration of all-trans retinoic acid. ${ }^{81}$

Relaxin-2, an endogenous hormone, has been reported with the properties to reduce fibrosis by inhibiting the pSmad2 signaling pathway. ${ }^{82,83}$ Mardhian et al ${ }^{84}$ utilized a nanosystem to deliver relaxin-2 to the pancreatic tumor model, observing a significant tumor inhibition by reducing the collagen expression, thus also improving gemcitabine efficacy. A recent study from $\mathrm{Hu}$ et $\mathrm{al}^{85}$ demonstrated the delivery of relaxin gene to the pancreatic tumor model, ameliorating the stroma environment and significantly inhibiting progression of metastasis to the liver.

Using miRNA to modulate the activated PSCs also provides a possible target therapy in pancreatic tumors, as miRNA can act as a tumor-promoter or suppressor. ${ }^{86}$ Kadera et $\mathrm{al}^{87}$ reported an overexpression of miRNA-21 in PDAC tumor associated fibroblasts, and this high expression significantly correlates with the poor overall survival. Sicard et al $^{88}$ demonstrated that depletion of miRNA-21 strongly inhibited PDAC tumor progression in vivo. MiR200 has been reported to be a regulator in reprogramming CAFs. CAFs showed an enhanced migration and invasion when down-regulating miRNA-200 in fibroblasts. ${ }^{89} \mathrm{~A}$ recent study from Vanessa et $\mathrm{al}^{90}$ indicated that the
miRNA-200 family can act as a tumor suppressor gene, suggesting the potential therapy target in pancreatic tumor treatment. Another study from our group reported miRNA199a and -214 to be overexpressed in activated PSCs and patient-derived pancreatic CAFs. ${ }^{91}$ We observed a significant reduction of migration, proliferation, and collagen expression in these PSCs after treating with the miRNA inhibitors $^{92}$ or anti-microRNA. ${ }^{93}$

Lee et $\mathrm{al}^{94}$ designed a urokinase plasminogen activator receptor (UPAR)-targeted magnetic iron oxide nanoparticle with gemcitabine loaded system showing uPAR-expressing tumor and stromal cells targeted delivery in pancreatic tumors. A significant inhibition of tumor growth was observed as a result of improved uptake of gemcitabine by the tumor tissue. ${ }^{94}$ Another strategy was reported by Chen et $\mathrm{al}^{95}$ in which they developed a two-step sequential delivery system to enhance pancreatic cancer therapy. First, they delivered the nitric oxide (NO) donor Snitroso-N-acetylpenicillamine (SNAP) loaded liposomes to PSCs to inhibit the production of dense stroma and subsequently administered gemcitabine loaded liposomes (Lip-GEM). This sequential approach led to enhanced intratumoral penetration of Lip-GEM due to the stromal disruption and enhanced efficacy, demonstrated in both a subcutaneous and orthotopic tumor mouse model.

Taking together, targeting CAFs is a promising strategy to inhibit CAF-induced desmoplasia barrier and thereby enhance the penetration of nanomedicine into PDAC.

\section{Targeting the Immune Cells in Pancreatic Cancer}

Macrophages and other immune cells such as T-cells, NK cells, and neutrophil are the main inflammatory cells infiltrating in the tumor tissues, in which these macrophages can be polarized to either anti-tumorigenic M1 phenotype or pro-tumorigenic M2 phenotype. ${ }^{42,96,97}$ M1 macrophages can generate a high level of proinflammatory cytokines such as IL-12 and IL-23, promoting leukocytes recruitment and activation to against tumor growth. ${ }^{98} \mathrm{M} 2$ macrophages, the most common type of tumor associated macrophages (TAMs), produce cytokines including TGF- $\beta$ and vascular endothelial growth factor, and promote the progression and metastasis of tumor by enhancing angiogenesis, immune suppression, and tumor cell invasion. ${ }^{99,100}$ Modulation of Thus, target to TAMs could be a possible strategy to improve pancreatic cancer treatment. 
Lipoxin, a family of bioactive lipid mediators locally secreted by immune cells, has been reported to have ability in anti-inflammation and anti-carcinogenesis. ${ }^{101}$ Zhou et $\mathrm{al}^{102}$ showed that Lipoxin A4 (LXA4) exhibited a protection effect in the acute pancreatitis model by reducing the pro-inflammatory cytokines as a result of inhibition of the NF- $\mathrm{KB}$ signaling pathway. Zong et $\mathrm{al}^{103}$ revealed that LXA4 inhibited pancreatic cancer cell invasion by suppressing the ROS/ERK/MMPs pathway. In addition, a following study from the same group demonstrated that LXA4 could reverse the mesenchymal phenotype of pancreatic tumor cells, thus suppressing the invasion and metastasis of tumor, on the basis of down-regulating TGF- $\beta 1$ signaling. ${ }^{104}$ Simoes et $\mathrm{al}^{105}$ tested ATL-1, an analog of LXA4, and found that ATL-1 inhibited tumor growth by stimulating TAM from M2- to M1-like profile change, thus inducing tumor cell apoptosis. In a follow-up study, they further investigated the mechanism of lipoxin anti-tumor profile, and demonstrated that ATL-1 reduced the TAMprecursor monocyte population compared to the control group and attenuated the M2 markers expression on TAMs, impairing the progression of tumor. ${ }^{106}$

Besides the lipoxins, others targeting the inflammation in the tumor may also improve tumoral drug delivery. ${ }^{107}$ Inflammation induces the increased extracellular fluid accumulation (known as edema) in the tumor, which hinders the effective intratumoral drug delivery. ${ }^{107}$ Cui et al ${ }^{108}$ performed a detailed metaanalysis of aspirin intake and found that, compared to low dose, high-dose aspirin intake might be correlated with the reduced risk of pancreatic cancer. It is suggested that non-steroidal anti-inflammatory drugs (NSAIDs) may suppress cyclooxygenases (COX) to inhibit vascular hyperpermeability, thus facilitating drug delivery in the tumor. ${ }^{107}$ Studies have shown the overexpression of COX observed in pancreatic cancer.109-112 A recent study from Ceponyte et $\mathrm{al}^{113}$ has compared NSAIDs activities in pancreatic cancer culture. They found that fenamates, coxibs, and salicylates showed significant cancer cell growth inhibition. Another study from Lyons et $\mathrm{al}^{114}$ demonstrated a reduction of collagen fibrillogenesis as well as inhibited cell migration in a breast cancer model after treatment with NSAIDs.

Taking together, this evidence suggests the potential benefit of targeting immune cells in pancreatic cancer treatment to reduce tumor stroma and enhance penetration.

\section{Targeting the Vasculature System in Pancreatic Cancer}

The tumor vasculature system mainly consists of endothelial cells and pericytes, which play a crucial role in the tumor development, progression, and metastasis. It has been recognized that angiogenesis promotes not only the growth of the tumor by delivering nutrients, but also the progression towards a more malignant and invasive tumor phenotype. Inhibiting the formation of vessels is, therefore, supposed to attenuate the progression of tumor. Early studies showed a high level of vascular endothelial growth factor (VEGF) production in PDAC as a result of angiogenesis. ${ }^{115}$ Anti-VEGF treatment has shown the capability of decreasing tumor growth and prolonging the survival of many cancer patients. ${ }^{116}$ However, many clinical studies showed frustrating results that anti-angiogenesis therapy tended to cause an increased invasion and metastasis profile on tumors. ${ }^{117-119}$ As the induced highly hypoxic tumor microenvironment stimulates tumor cells to escape by promoting EMT (epithelial-to-mesenchymaltransformation) which leads to a more flexible phenotype on tumor cells. ${ }^{120}$ Besides that, anti-angiogenic agents may also damage the integrity of the vessels, reduce the coverage of pericyte and decrease microvessel density, thus facilitating the intravasation of cancer cells. ${ }^{121}$ Therefore, the use of bevacizumab, an approved monoclonal antibody of VEGF, in combination with gemcitabine failed to show benefit in overall survival in pancreatic cancer patients in the clinical phase III study. ${ }^{122}$ A similar phase I/II study also confirmed that multiple chemotherapeutics with bevacizumab did not improve efficacy in metastatic pancreatic cancer patients. ${ }^{123}$ However, a recent study from Shen et al $^{124}$ reported that using renin-angiotensin inhibitor in combination with bevacizumab significantly prolonged the survival in metastatic colorectal cancer patients.

The tumor vasculature system is not only crucial for delivering nutrients but also therapeutics and therefore is a pivotal component controlling the effect of therapeutics. In pancreatic tumors, the tumor vasculature is often collapsed, distorted, and poorly functional due to abundant stroma, high intratumoral pressure, leading to poor tumor perfusion, and insufficient intratumor drug delivery into the tumor. ${ }^{125-127}$ Major strategies to improve tumor perfusion have reduced desmoplasia using CAF-targeting approaches, as discussed above. Another approach is the vessel normalization which has been suggested to improve 
the chemotherapy perfusion and efficacy. ${ }^{128,129}$ Nagathihalli et $\mathrm{al}^{130}$ showed that inhibition of STAT3 (signal transducer and activator of transcription 3) combined with gemcitabine effectively normalized the vasculature of pancreatic tumor by attenuating the expression of osteonectin and increasing microvessel density, exhibiting an improved chemotherapeutics delivery into the tumor and thereby improved tumor inhibition. Kim et al used HS-173, the phosphoinositide 3-kinase inhibitor to normalized pancreatic tumor vessels by inhibiting vascular leakage and neoangiogenesis. With this method, they observed an increased perfusion and improved therapeutic response when co-administrating with doxorubicin. ${ }^{131} \mathrm{~A}$ study from Trindade et $\mathrm{al}^{132}$ reported that increasing Dll4/ Notch signaling decreased the VEGF-induced endothelial proliferation and improved the maturation functionality of tumor vessels, thus reducing the metastasis and promoting the drug delivery. Vessel normalization can also benefit the immune effector cells infiltration to the tumor. Hamzah et $\mathrm{al}^{133}$ inhibited the regulator of G-protein signaling 5, inducing vascular normalization in a pancreatic islet cancer model. A more uniformly distributed and less leaky vasculature was observed, facilitating immune cell infiltration and significantly improving the survival of the model mice.

Improvement of tumor blood perfusion is one of the strategies to resolve tumor-associated hypoperfusion hindering nanoparticle penetration. Wei et $\mathrm{al}^{134}$ developed doxorubicin-loaded liposomes that carried low-density cilengitide, an $\alpha v \beta 3$ integrin-specific cyclic peptide, via a membrane type 1-matrix metalloproteinase (MT1-MMP) cleavable peptide. In vivo, cilengitide, at a low dose showed the proangiogenic activity through cleavage by MT1-MMP on tumor endothelial cells and increased tumor blood perfusion, thereby improving the tumor accumulation and distribution of doxorubicin-loaded liposomes. These liposomes then displayed enhanced penetration and increased cellular uptake upon heat-triggered release, leading to the improved efficacy in vivo. Kuninty et al $^{14}$ applied AV3, which was an antagonist against integrin $\alpha 5$ to inhibit the ECM and fibroblast contraction induced by activation of CAFs, to treat pancreatic tumors followed by administration of gemcitabine, observing the decompression of blood vessels and thus improving intratumoral perfusion. This decompression facilitated the therapeutics perfusing into the tumor and produced higher drug uptake level, thus significantly inhibiting the tumor growth compared with other groups in these mouse tumor models.

\section{Targeting the Extracellular Matrix in Pancreatic Cancer}

The excess deposition of extracellular matrix (ECM) is a hallmark of pancreatic cancer. These ECM proteins and other components (eg, collagens, hyaluronic acid) in the tumor stroma form a physical barrier, increasing intratumoral pressure as well as contract the vessels, leading to limited drug delivery to the tumor. ${ }^{135}$ Evidence has shown that PDAC patients with high stromal hyaluronic acid exhibited much shorter overall survival compared to those with low hyaluronic acid. ${ }^{136}$ Similarly, patients with a high level of collagen expression showed a poor overall survival compared to those with low collagen levels. ${ }^{136,137}$ These studies indicate that excessive ECM deposition is negatively correlated with the patient outcome and therefore represents a crucial target. Targeting the ECM may provide new therapeutic strategies in PDAC treatment.

Studies have shown that hyaluronic acid degradation in pancreatic tumor stroma led to the normalization of interstitial fluid pressure and re-opening of collapsed vessels, thus improving drug delivery to the tumor. ${ }^{125,126}$ PEGylated hyaluronidase 20 (PEGPH20) has been formulated to improve hyaluronic acid degradation in vivo. ${ }^{138} \mathrm{In}$ a PDAC mouse model, Provenzano et $\mathrm{al}^{126}$ showed a substantial reduction in the tumor size when PEGPH20 was used in combination with gemcitabine attributed to enhanced drug delivery, thus observing a significant prolonged survival compared to gemcitabine alone. A similar study from Jacobetz et al, ${ }^{125}$ showed that administration of PEGPH20 reduced the vascular collapse and improved intratumoral uptake of gemcitabine. Given the effects observed in animal models, clinical studies were performed with PEGPH20. A phase Ib/II study showed a significant increase of perfusion in PDAC patient treated with PEGPH20 in tumors with high hyaluronic acid levels. ${ }^{139}$ Furthermore, PEGPH20-related adverse events such as musculoskeletal and extremity pain, peripheral edema, and thromboembolic events were reported in patients. Recently, a phase III study reported that PEGPH20, however, did not show improved outcome when co-administration with nab-paclitaxel/gemcitabine compared to chemotherapy alone (median overall survival: 
11.2 versus 11.5 months; median progression free survival: 7.1 versus 7.1 months). ${ }^{140}$

A study from $\mathrm{Ji}$ et $\mathrm{al}^{74}$ reported a MMP-2 responsive liposome with pirfenidone (PFD) encapsulated to downregulate the components of ECM first, followed by gemcitabine treatment. The result showed a clearly deeper penetration of drug after the liposomal PFD treatment, thus leading to improved tumor inhibition. ${ }^{74}$ Although degradation of collagen in stroma may also be a therapeutic approach to enhance drug delivery, there have been several studies reporting that lower stroma content may promote the development of PDAC. ${ }^{57,141}$ A recent study from Chen et al $^{142}$ also showed that deletion of type I collagen in myofibroblasts induced immune suppression and reduction of overall survival. The reason could, in part, be the excessive depletion of matrix proteins, which in turn facilitates the tumor cells invasion and dissemination. Another study from Zinger et al ${ }^{143}$ reported pretreatment of collagenase liposome to the pancreatic tumor allowed the follow-up drug penetration. They found that, based on this regimen, degrading the ECM facilitated paclitaxel micelles penetration and suppressed the tumor growth without affecting tumor cell metastasis. ${ }^{143}$

Altogether, these findings suggest that ECM can be an alternative target for pancreatic cancer treatment, but how to maximize this ECM-targeted therapy benefit with nanomedicine still needs more understanding. Probably reduction of ECM proteins in the tumor to some extent then followed by (nano)chemotherapy may achieve a better efficacy since it induces the deep drug penetration and uptake.

\section{Conclusion}

Poor perfusion and penetration of therapeutics into the PDAC tissue poses a great challenge to achieve an effective therapeutic response in patients. PDAC tissue has several barriers associated with the drug penetration due to the inherently developed compressed blood vasculature hindering tumor perfusion and excessive ECM deposition forming a physical barrier for deep penetration. These features not only limit the effective delivery of therapeutics and impede the efficacy, but also make this tumor highly aggressive and resistant to therapy. Besides the primary tumor, treatment of (micro)metastasis is a key feature for most advanced PDAC and is a serious problem. Tumor penetration into micrometastatic tumors is therefore an important issue to address. These micrometastatic tumors are poorly vascularized and difficult to access for small drug molecules and nanomedicine. To our knowledge there is no focused study to enhance drug penetration in metastatic tumors and most studies are dedicated to the primary tumor.

In efforts to enhance tumor penetration, both the nanomedicine field and tumor microenvironment fields have advanced technologies which are discussed herein. Nanomedicine technologies such as size reduction, triggered enhanced drug release, and tumor penetration via transcytosis are some of the reported strategies which are proven in vivo in PDAC models. Yet, these strategies are fundamentally confirmed and their clinical translation still needs to progress. On the other hand, different anti-stromal therapies developed during the last decade showing enhanced efficacy in experimental models were rushed to the clinical trials. Many of these strategies, however, resulted in clinical failures, either due to intolerable adverse effects or lack of efficacy. This opened up a debate about the "pro-tumorigenic" or "anti-tumorigenic" role of tumor stroma in PDAC. It is more likely that complete depletion of stroma (ECM or CAFs) is not an optimal solution which indicate towards re-programming of the tumor stroma.

Of note, in contrast to the many other tumor-associated mechanisms, our understanding on the role of stroma is still in its juvenile stage. In the last 5 years, several subtypes of CAFs and other roles of stroma have been identified and understood, which will enrich the therapeutic pipeline for developing new strategies to overcome stromal barriers. Furthermore, repurposing of drugs such as losartan, alltrans retinoic acid, etc., as reported in this review, will expedite the drug development against stroma barrier. Yet, new pathways are urgently needed to be explored to enhance the drug penetration in this hard-to-treat cancer. Furthermore, nanomedicine approaches provide great tools to combine different drugs either for co-delivery or sequential delivery of anti-stromal drugs and chemotherapeutic agents. We have increasingly seen many creative and innovative ideas from researchers to enhance the penetration of nanomedicine and also deliver complex molecules such as siRNA and microRNA. Yet, the challenge to bring these emerging technologies to clinic is massive and remains limited to the scientific publications. Overall, enhancement of drug penetration in the pancreatic tumor is a vital approach which needs clinical validation in future.

\section{Acknowledgments}

This project was supported by Top Technology Twente: Connecting Industry program (SBD 2018/013, funded to J.P.). 


\section{Disclosure}

The authors report no conflicts of interest in this work.

\section{References}

1. Rawla P, Sunkara T, Gaduputi V. Epidemiology of pancreatic cancer: global trends, etiology and risk factors. World $J$ Oncol. 2019;10(1):10. doi:10.14740/wjon1166

2. Sung H, Ferlay J, Siegel RL, et al. Global cancer statistics 2020: GLOBOCAN estimates of incidence and mortality worldwide for 36 cancers in 185 countries. CA Cancer J Clin. 2021;1:2.

3. Pelosi E, Castelli G, Testa U. Pancreatic cancer: molecular characterization, clonal evolution and cancer stem cells. Biomedicines. 2017;5(4):65. doi:10.3390/biomedicines5040065

4. Bray F, Ferlay J, Soerjomataram I, Siegel RL, Torre LA, Jemal A. Global cancer statistics 2018: GLOBOCAN estimates of incidence and mortality worldwide for 36 cancers in 185 countries. CA Cancer J Clin. 2018;68(6):394-424.

5. Ferlay J, Partensky C, Bray F. More deaths from pancreatic cancer than breast cancer in the EU by 2017. Acta Oncologica. 2016;55(9-10):1158-1160. doi:10.1080/0284186X.2016.1197419

6. Patra CR, Bhattacharya R, Mukhopadhyay D, Mukherjee P. Fabrication of gold nanoparticles for targeted therapy in pancreatic cancer. Adv Drug Deliv Rev. 2010;62(3):346-361. doi:10.1016/j.addr.2009.11.007

7. Manji GA, Olive KP, Saenger YM, Oberstein P. Current and emerging therapies in metastatic pancreatic cancer. Clin Cancer Res. 2017;23 (7):1670-1678. doi:10.1158/1078-0432.CCR-16-2319

8. Lei F, Xi X, Batra SK, Bronich TK. Combination therapies and drug delivery platforms in combating pancreatic cancer. J Pharmacol Exp Therapeutics. 2019;370(3):682-694. doi:10.1124/jpet.118.255786

9. Gresham GK, Wells GA, Gill S, Cameron C, Jonker DJ. Chemotherapy regimens for advanced pancreatic cancer: a systematic review and network meta-analysis. BMC Cancer. 2014;14 (1):1-13. doi:10.1186/1471-2407-14-471

10. Aprile G, Negri FV, Giuliani F, et al. Second-line chemotherapy for advanced pancreatic cancer: which is the best option? Crit Rev Oncol Hematol. 2017;115:1-12. doi:10.1016/j.critrevonc.2017.03.025

11. Hidalgo M, Cascinu S, Kleeff J, et al. Addressing the challenges of pancreatic cancer: future directions for improving outcomes. Pancreatology. 2015;15(1):8-18. doi:10.1016/j.pan.2014.10.001

12. O'neil B, Scott A, Ma WW, et al. A phase II/III randomized study to compare the efficacy and safety of rigosertib plus gemcitabine versus gemcitabine alone in patients with previously untreated metastatic pancreatic cancer. Ann Oncol. 2015;26(9):1923-1929. doi:10.1093/annonc/mdv264

13. Erkan M, Hausmann S, Michalski CW, et al. The role of stroma in pancreatic cancer: diagnostic and therapeutic implications. Nat Rev Gastroenterol Hepatol. 2012;9(8):454. doi:10.1038/ nrgastro.2012.115

14. Kuninty PR, Bansal R, De Geus SW, et al. ITGA5 inhibition in pancreatic stellate cells attenuates desmoplasia and potentiates efficacy of chemotherapy in pancreatic cancer. Sci Adv. 2019;5 (9):eaax2770. doi:10.1126/sciadv.aax2770

15. Chu GC, Kimmelman AC, Hezel AF, DePinho RA. Stromal biology of pancreatic cancer. $J$ Cell Biochem. 2007;101(4):887907. doi:10.1002/jcb. 21209

16. McCarroll JA, Naim S, Sharbeen G, et al. Role of pancreatic stellate cells in chemoresistance in pancreatic cancer. Front Physiol. 2014;5:141. doi:10.3389/fphys.2014.00141

17. Adiseshaiah PP, Crist RM, Hook SS, McNeil SE. Nanomedicine strategies to overcome the pathophysiological barriers of pancreatic cancer. Nat Rev Clin Oncol. 2016;13(12):750. doi:10.1038/ nrclinonc.2016.119
18. Li J, Kataoka K. Chemo-physical strategies to advance the in vivo functionality of targeted nanomedicine: the next generation. $\mathrm{J} \mathrm{Am}$ Chem Soc. 2020;143(2):538-559. doi:10.1021/jacs.0c09029

19. Park J, Choi Y, Chang H, Um W, Ryu JH, Kwon IC. Alliance with EPR effect: combined strategies to improve the EPR effect in the tumor microenvironment. Theranostics. 2019;9(26):8073. doi:10.7150/thno. 37198

20. Wang AZ. EPR or no EPR? The billion-dollar question. Sci Transl Med. 2015;7(294):294ec112-294ec112. doi:10.1126/scitranslmed.aac 8108

21. Von Hoff DD, Ervin T, Arena FP, et al. Increased survival in pancreatic cancer with nab-paclitaxel plus gemcitabine. $N$ Eng $J$ Med. 2013;369(18):1691-1703. doi:10.1056/NEJMoa1304369

22. Wang-Gillam A, Li C-P, Bodoky G, et al. Nanoliposomal irinotecan with fluorouracil and folinic acid in metastatic pancreatic cancer after previous gemcitabine-based therapy (NAPOLI-1): a global, randomised, open-label, Phase 3 trial. Lancet. 2016;387 (10018):545-557. doi:10.1016/S0140-6736(15)00986-1

23. Kalra AV, Kim J, Klinz SG, et al. Preclinical activity of nanoliposomal irinotecan is governed by tumor deposition and intratumor prodrug conversion. Cancer Res. 2014;74(23):7003-7013. doi:10.1158/0008-5472.CAN-14-0572

24. Wang H, Mislati R, Ahmed R, et al. Elastography can map the local inverse relationship between shear modulus and drug delivery within the pancreatic ductal adenocarcinoma microenvironment. Clin Cancer Res. 2019;25(7):2136-2143. doi:10.1158/ 1078-0432.CCR-18-2684

25. Nia HT, Munn LL, Jain RK. Mapping physical tumor microenvironment and drug delivery. Clin Cancer Res. 2019;25(7):20242026. doi:10.1158/1078-0432.CCR-18-3724

26. Li H-J, Du J-Z, Liu J, et al. Smart superstructures with ultrahigh $\mathrm{pH}$-sensitivity for targeting acidic tumor microenvironment: instantaneous size switching and improved tumor penetration. ACS Nano. 2016;10(7):6753-6761. doi:10.1021/acsnano.6b02326

27. Cun X, Chen J, Li M, et al. Tumor-associated fibroblast-targeted regulation and deep tumor delivery of chemotherapeutic drugs with a multifunctional size-switchable nanoparticle. ACS Appl Mater Interfaces. 2019;11(43):39545-39559. doi:10.1021/acsa mi.9b13957

28. Li J, Han Y, Chen Q, et al. Dual endogenous stimuli-responsive polyplex micelles as smart two-step delivery nanocarriers for deep tumor tissue penetration and combating drug resistance of cisplatin. J Mater Chem B. 2014;2(13):1813-1824. doi:10.1039/ C3TB21383H

29. Li J, Ke W, Li H, Zha Z, Han Y, Ge Z. Endogenous stimuli-sensitive multistage polymeric micelleplex anticancer drug delivery system for efficient tumor penetration and cellular internalization. $A d v$ Healthcare Mater. 2015;4(15):2206-2219. doi:10.1002/adhm.2 01500379

30. Li J, Wei K, Zuo S, et al. Light-Triggered Clustered Vesicles with Self-Supplied Oxygen and Tissue Penetrability for Photodynamic Therapy against Hypoxic Tumor. Adv Funct Mater. 2017;27 (33):1702108. doi:10.1002/adfm.201702108

31. Sun J, Wan Z, Chen Y, et al. Triple drugs co-delivered by a small gemcitabine-based carrier for pancreatic cancer immunochemotherapy. Acta biomaterialia. 2020;106:289-300. doi:10.1016/j. actbio.2020.01.039

32. Lu T, Lokerse WJ, Seynhaeve AL, Koning GA, Ten Hagen TL. Formulation and optimization of idarubicin thermosensitive liposomes provides ultrafast triggered release at mild hyperthermia and improves tumor response. $J$ Controlled Release. 2015;220:425-437. doi:10.1016/j.jconrel.2015.10.056

33. Lu T, Haemmerich D, Liu H, et al. Externally triggered smart drug delivery system encapsulating idarubicin shows superior kinetics and enhances tumoral drug uptake and response. Theranostics. 2021;11(12):5700. doi:10.7150/thno.55163 
34. Li L, Hagen TL, Hossann M, et al. Mild hyperthermia triggered doxorubicin release from optimized stealth thermosensitive liposomes improves intratumoral drug delivery and efficacy. $J$ Controlled Release. 2013;168(2):142-150. doi:10.1016/j.jconrel.2013.03.011

35. Needham D, Anyarambhatla G, Kong G, Dewhirst MW. A new temperature-sensitive liposome for use with mild hyperthermia: characterization and testing in a human tumor xenograft model. Cancer Res. 2000;60(5):1197-1201.

36. Malekigorji M, Alfahad M, Lin PKT, Jones S, Curtis A, Hoskins C. Thermally triggered theranostics for pancreatic cancer therapy. Nanoscale. 2017;9(34):12735-12745. doi:10.1039/C7NR02751F

37. Emamzadeh M, Desmaële D, Couvreur P, Pasparakis G. Dual controlled delivery of squalenoyl-gemcitabine and paclitaxel using thermo-responsive polymeric micelles for pancreatic cancer. J Mater Chem B. 2018;6(15):2230-2239. doi:10.1039/C7TB02899G

38. Lo JH, Hao L, Muzumdar MD, et al. iRGD-guided tumor-penetrating nanocomplexes for therapeutic siRNA delivery to pancreatic cancer. Mol Cancer Ther. 2018;17(11):2377-2388. doi:10.1158/1535-7163.MCT-17-1090

39. Wang G, Zhou Z, Zhao Z, et al. Enzyme-Triggered Transcytosis of Dendrimer-Drug Conjugate for Deep Penetration into Pancreatic Tumors. ACS Nano. 2020;14(4):4890-4904. doi:10.1021/acsnano.0c00974

40. Chen X, Zhou W, Liang C, et al. Codelivery nanosystem targeting the deep microenvironment of pancreatic cancer. Nano Lett. 2019;19(6):3527-3534. doi:10.1021/acs.nanolett.9b00374

41. Hanahan D, Coussens LM. Accessories to the crime: functions of cells recruited to the tumor microenvironment. Cancer Cell. 2012;21(3):309-322. doi:10.1016/j.ccr.2012.02.022

42. Mueller MM, Fusenig NE. Friends or foes - bipolar effects of the tumour stroma in cancer. Nat Rev Cancer. 2004;4(11):839-849. doi:10.1038/nrc1477

43. Schnittert J, Bansal R, Prakash J. Targeting pancreatic stellate cells in cancer. Trends Cancer. 2019;5(2):128-142. doi:10.1016/j. trecan.2019.01.001

44. Biffi G. Tracing the Origin of Fibroblasts in Pancreatic Cancer. Cell Mol Gastroenterol Hepatol. 2020;10(3):645. doi:10.1016/j. jcmgh.2020.06.008

45. Öhlund D, Handly-Santana A, Biffi G, et al. Distinct populations of inflammatory fibroblasts and myofibroblasts in pancreatic cancer. J Exp Med. 2017;214(3):579-596. doi:10.1084/jem.20162024

46. Elyada E, Bolisetty M, Laise P, et al. Cross-species single-cell analysis of pancreatic ductal adenocarcinoma reveals antigenpresenting cancer-associated fibroblasts. Cancer Discov. 2019;9 (8):1102-1123. doi:10.1158/2159-8290.CD-19-0094

47. Apte MV, Wilson JS, Lugea A, Pandol SJ. A starring role for stellate cells in the pancreatic cancer microenvironment. Gastroenterology. 2013;144(6):1210-1219. doi:10.1053/j.gastro.2012.11.037

48. Liu H, Shi Y, Qian F. Opportunities and delusions regarding drug delivery targeting pancreatic cancer-associated fibroblasts. $A d v$ Drug Deliv Rev. 2021.

49. Walter K, Omura N, Hong S-M, et al. Overexpression of smoothened activates the sonic hedgehog signaling pathway in pancreatic cancer-associated fibroblasts. Clin Cancer Res. 2010;16(6):17811789. doi:10.1158/1078-0432.CCR-09-1913

50. Bailey JM, Swanson BJ, Hamada T, et al. Sonic hedgehog promotes desmoplasia in pancreatic cancer. Clin Cancer Res. 2008;14(19):5995-6004. doi:10.1158/1078-0432.CCR-08-0291

51. Bailey JM, Mohr AM, Hollingsworth MA. Sonic hedgehog paracrine signaling regulates metastasis and lymphangiogenesis in pancreatic cancer. Oncogene. 2009;28(40):3513-3525. doi:10.1038/onc. 2009.220

52. Olive KP, Jacobetz MA, Davidson CJ, et al. Inhibition of Hedgehog signaling enhances delivery of chemotherapy in a mouse model of pancreatic cancer. Science. 2009;324 (5933):1457-1461. doi:10.1126/science.1171362
53. Amakye D, Jagani Z, Dorsch M. Unraveling the therapeutic potential of the Hedgehog pathway in cancer. Nat Med. 2013;19 (11):1410. doi:10.1038/nm.3389

54. Lee JJ, Perera RM, Wang H, et al. Stromal response to Hedgehog signaling restrains pancreatic cancer progression. Proce Nat Acad Sci. 2014;111(30):E3091-E3100. doi:10.1073/pnas.1411679111

55. Rhim AD, Oberstein PE, Thomas DH, et al. Stromal elements act to restrain, rather than support, pancreatic ductal adenocarcinoma. Cancer Cell. 2014;25(6):735-747. doi:10.1016/j.ccr.2014.04.021

56. De Jesus-acosta A, Sugar EA, O'Dwyer PJ, et al. Phase 2 study of vismodegib, a hedgehog inhibitor, combined with gemcitabine and nab-paclitaxel in patients with untreated metastatic pancreatic adenocarcinoma. Br J Cancer. 2020;122(4):498-505. doi:10.10 38/s41416-019-0683-3

57. Özdemir BC, Pentcheva-Hoang T, Carstens JL, et al. Depletion of carcinoma-associated fibroblasts and fibrosis induces immunosuppression and accelerates pancreas cancer with reduced survival. Cancer Cell. 2015;28(6):831-833. doi:10.1016/j.ccell.2015.11.002

58. Prakash J. Cancer-associated fibroblasts: perspectives in cancer therapy. Trends Cancer. 2016;2(6):277-279. doi:10.1016/j. trecan.2016.04.005

59. Hynes RO. Integrins: bidirectional, allosteric signaling machines. cell. 2002;110(6):673-687. doi:10.1016/S0092-8674(02)00971-6

60. Schnittert J, Bansal R, Storm G, Prakash J. Integrins in wound healing, fibrosis and tumor stroma: high potential targets for therapeutics and drug delivery. Adv Drug Deliv Rev. 2018;129:37-53. doi:10.1016/j.addr.2018.01.020

61. Schnittert J, Bansal R, Mardhian DF, Van Baarlen J, Östman A, Prakash J. Integrin $\alpha 11$ in pancreatic stellate cells regulates tumor stroma interaction in pancreatic cancer. FASEB J. 2019;33 (5):6609-6621. doi:10.1096/fj.201802336R

62. Charrier A, Brigstock DR. Regulation of pancreatic function by connective tissue growth factor (CTGF, CCN2). Cytokine Growth Factor Rev. 2013;24(1):59-68. doi:10.1016/j.cytogfr.2012.07.001

63. Lali FV, Martin YH, Metcalfe AD. Advances in biopharmaceutical agents and growth factors for wound healing and scarring. Skin Tissue Eng Regen Med. 2016;14:337-355.

64. Aikawa T, Gunn J, Spong SM, Klaus SJ, Korc M. Connective tissue growth factor-specific antibody attenuates tumor growth, metastasis, and angiogenesis in an orthotopic mouse model of pancreatic cancer. Mol Cancer Ther. 2006;5(5):1108-1116. doi:10.1158/1535-7163.MCT-05-0516

65. Nevala-Plagemann C, Hidalgo M, Garrido-Laguna I. From stateof-the-art treatments to novel therapies for advanced-stage pancreatic cancer. Nat Rev Clin Oncol. 2020;17(2):108-123. doi:10.1038/s41571-019-0281-6

66. Resovi A, Borsotti P, Ceruti T, et al. CCN-based therapeutic peptides modify pancreatic ductal adenocarcinoma microenvironment and decrease tumor growth in combination with chemotherapy. Cells. 2020;9(4):952. doi:10.3390/cells9040952

67. Han H, Valdepérez D, Jin Q, et al. Dual enzymatic reactionassisted gemcitabine delivery systems for programmed pancreatic cancer therapy. Acs Nano. 2017;11(2):1281-1291. doi:10.1021/ acsnano.6b05541

68. Han H, Hou Y, Chen X, et al. Metformin-induced stromal depletion to enhance the penetration of gemcitabine-loaded magnetic nanoparticles for pancreatic cancer targeted therapy. J Am Chem Soc. 2020;142(10):4944-4954. doi:10.1021/jacs.0c00650

69. Duan W, Chen K, Jiang Z, et al. Desmoplasia suppression by metformin-mediated AMPK activation inhibits pancreatic cancer progression. Cancer Lett. 2017;385:225-233. doi:10.1016/j. canlet.2016.10.019

70. Ilmer M, Westphalen CB, Niess H, et al. Repurposed drugs in pancreatic ductal adenocarcinoma: an update. Cancer J. 2019;25 (2):134-138. doi:10.1097/PPO.0000000000000372 
71. Polydorou C, Mpekris F, Papageorgis P, Voutouri C, Stylianopoulos T. Pirfenidone normalizes the tumor microenvironment to improve chemotherapy. Oncotarget. 2017;8(15):24506. doi:10.18632/oncotarget.15534

72. Kozono S, Ohuchida K, Eguchi D, et al. Pirfenidone inhibits pancreatic cancer desmoplasia by regulating stellate cells. Cancer Res. 2013;73(7):2345-2356. doi:10.1158/0008-5472.CAN-12-3180

73. Gao F, Wu J, Niu S, et al. Biodegradable, $\mathrm{pH}$-sensitive hollow mesoporous organosilica nanoparticle (HMON) with controlled release of pirfenidone and ultrasound-target-microbubble-destruction (UTMD) for pancreatic cancer treatment. Theranostics. 2019;9(20):6002. doi:10.7150/thno.36135

74. Ji T, Lang J, Wang J, et al. Designing liposomes to suppress extracellular matrix expression to enhance drug penetration and pancreatic tumor therapy. ACS Nano. 2017;11(9):8668-8678. doi:10.1021/acsnano.7b01026

75. Senoo H, Mezaki Y, Fujiwara M. The stellate cell system (vitamin A-storing cell system). Anat Sci Int. 2017;92(4):387-455.

76. Froeling FE, Feig $\mathrm{C}$, Chelala $\mathrm{C}$, et al. Retinoic acid-induced pancreatic stellate cell quiescence reduces paracrine Wnt- $\beta$-catenin signaling to slow tumor progression. Gastroenterology. 2011;141(4):1486-1497. doi:10.1053/j.gastro.2011.06.047

77. Jaster R, Hilgendorf I, Fitzner B, et al. Regulation of pancreatic stellate cell function in vitro: biological and molecular effects of all-trans retinoic acid. Biochem Pharmacol. 2003;66(4):633-641. doi:10.1016/S0006-2952(03)00390-3

78. Carapuça EF, Gemenetzidis E, Feig C, et al. Anti-stromal treatment together with chemotherapy targets multiple signalling pathways in pancreatic adenocarcinoma. J Pathol. 2016;239(3):286296. doi:10.1002/path.4727

79. Ene-Obong A, Clear AJ, Watt J, et al. Activated pancreatic stellate cells sequester CD8 $+\mathrm{T}$ cells to reduce their infiltration of the juxtatumoral compartment of pancreatic ductal adenocarcinoma. Gastroenterology. 2013;145(5):1121-1132. doi:10.1053/j. gastro.2013.07.025

80. Han X, Li Y, Xu Y, et al. Reversal of pancreatic desmoplasia by re-educating stellate cells with a tumour microenvironment-activated nanosystem. Nat Commun. 2018;9(1):1-18. doi:10.1038/ s41467-018-05906-x

81. Kocher HM, Basu B, Froeling FE, et al. Phase I clinical trial repurposing all-trans retinoic acid as a stromal targeting agent for pancreatic cancer. Nat Commun. 2020;11(1):1-9. doi:10.1038/ s41467-020-18636-w

82. Samuel CS, Bodaragama H, Chew JY, Widdop RE, Royce SG, Hewitson TD. Serelaxin is a more efficacious antifibrotic than enalapril in an experimental model of heart disease. Hypertension. 2014;64(2):315-322.

doi:10.1161/ HYPERTENSIONAHA.114.03594

83. Samuel CS, Unemori EN, Mookerjee I, et al. Relaxin modulates cardiac fibroblast proliferation, differentiation, and collagen production and reverses cardiac fibrosis in vivo. Endocrinology. 2004;145(9):4125-4133. doi:10.1210/en.2004-0209

84. Mardhian DF, Storm G, Bansal R, Prakash J. Nano-targeted relaxin impairs fibrosis and tumor growth in pancreatic cancer and improves the efficacy of gemcitabine in vivo. $J$ Controlled Release. 2018;290:1-10. doi:10.1016/j.jconrel.2018.09.031

85. $\mathrm{Hu}$ M, Wang Y, Xu L, et al. Relaxin gene delivery mitigates liver metastasis and synergizes with check point therapy. Nat Commun. 2019;10(1):1-13. doi:10.1038/s41467-019-10893-8

86. Kuninty PR, Schnittert J, Storm G, Prakash J. MicroRNA targeting to modulate tumor microenvironment. Front Oncol. 2016;6:3. doi:10.3389/fonc. 2016.00003

87. Kadera BE, Li L, Toste PA, et al. MicroRNA-21 in pancreatic ductal adenocarcinoma tumor-associated fibroblasts promotes metastasis. PLoS One. 2013;8(8):e71978. doi:10.1371/journal. pone. 0071978
88. Sicard F, Gayral M, Lulka H, Buscail L, Cordelier P. Targeting miR-21 for the therapy of pancreatic cancer. Mol Therapy. 2013;21(5):986-994. doi:10.1038/mt.2013.35

89. Tang X, Hou Y, Yang G, et al. Stromal miR-200s contribute to breast cancer cell invasion through CAF activation and ECM remodeling. Cell Death Differ. 2016;23(1):132-145. doi:10.10 38/cdd.2015.78

90. Diaz-Riascos ZV, Ginesta MM, Fabregat J, et al. Expression and role of microRNAs from the miR-200 family in the tumor formation and metastatic propensity of pancreatic cancer. Mol Therapy Nucleic Acids. 2019;17:491-503. doi:10.1016/j. omtn.2019.06.015

91. Kota J, Hancock J, Kwon J, Korc M. Pancreatic cancer: stroma and its current and emerging targeted therapies. Cancer Lett. 2017;391:38-49. doi:10.1016/j.canlet.2016.12.035

92. Kuninty PR, Bojmar L, Tjomsland V, et al. MicroRNA-199a and214 as potential therapeutic targets in pancreatic stellate cells in pancreatic tumor. Oncotarget. 2016;7(13):16396. doi:10.18632/ oncotarget.7651

93. Schnittert J, Kuninty PR, Bystry TF, Brock R, Storm G, Prakash J. Anti-microRNA targeting using peptide-based nanocomplexes to inhibit differentiation of human pancreatic stellate cells. Nanomedicine. 2017;12(12):1369-1384. doi:10.2217/nnm-20170054

94. Lee GY, Qian WP, Wang L, et al. Theranostic nanoparticles with controlled release of gemcitabine for targeted therapy and MRI of pancreatic cancer. ACS Nano. 2013;7(3):2078-2089. doi:10.1021/ nn3043463

95. Chen X, Jia F, Li Y, et al. Nitric oxide-induced stromal depletion for improved nanoparticle penetration in pancreatic cancer treatment. Biomaterials. 2020;246:119999. doi:10.1016/j.biomateri als.2020.119999

96. De Visser KE, Eichten A, Coussens LM. Paradoxical roles of the immune system during cancer development. Nat Rev Cancer. 2006;6(1):24-37. doi:10.1038/nrc1782

97. Jayasingam SD, Citartan M, Thang TH, Mat Zin AA, Ang KC, Ch'ng ES. Evaluating the polarization of tumor-associated macrophages into M1 and M2 phenotypes in human cancer tissue: technicalities and challenges in routine clinical practice. Front Oncol. 2020;9:1512. doi:10.3389/fonc.2019.01512

98. Ngambenjawong C, Gustafson HH, Pun SH. Progress in tumorassociated macrophage (TAM)-targeted therapeutics. Adv Drug Deliv Rev. 2017;114:206-221. doi:10.1016/j.addr.2017.04.010

99. Yang L, Zhang Y. Tumor-associated macrophages: from basic research to clinical application. J Hematol Oncol. 2017;10(1):112. doi:10.1186/s13045-017-0430-2

100. Lin $\mathrm{Y}, \mathrm{Xu} \mathrm{J}$, Lan H. Tumor-associated macrophages in tumor metastasis: biological roles and clinical therapeutic applications. $J$ Hematol Oncol. 2019;12(1):1-16. doi:10.1186/s13045-0190760-3

101. Zhang T, Hao H, Zhou X-Y. The role of lipoxin in regulating tumor immune microenvironments. Prostaglandins Other Lipid Mediat. 2019;144:106341. doi:10.1016/j.prostaglandins.20 19.106341

102. Zhou M, Chen B, Sun H, Deng Z, Andersson R, Zhang Q. The protective effects of Lipoxin A4 during the early phase of severe acute pancreatitis in rats. Scand J Gastroenterol. 2011;46(2):211219. doi:10.3109/00365521.2010.525715

103. Zong L, Li J, Chen X, et al. Lipoxin A4 attenuates cell invasion by inhibiting ROS/ERK/MMP pathway in pancreatic cancer. Oxid Med Cell Longev. 2016;2:2016.

104. Zong L, Chen K, Jiang Z, et al. Lipoxin A4 reverses mesenchymal phenotypes to attenuate invasion and metastasis via the inhibition of autocrine TGF- $\beta 1$ signaling in pancreatic cancer. $J$ Exp Clin Cancer Res. 2017;36(1):1-12. doi:10.1186/s13046-0170655-5 
105. Simoes R, De-Brito N, Cunha-Costa H, et al. Lipoxin A4 selectively programs the profile of M2 tumor-associated macrophages which favour control of tumor progression. Int $j$ Cancer. 2017;140(2):346-357. doi:10.1002/ijc.30424

106. De-brito NM, Da-costa HC, Simões RL, Barja-Fidalgo C. Lipoxin-Induced Phenotypic Changes in CD115+ LY6Chi Monocytes TAM Precursors Inhibits Tumor Development. Front Oncol. 2019;9:540. doi:10.3389/fonc.2019.00540

107. Gkretsi V, Zacharia LC, Stylianopoulos T. Targeting inflammation to improve tumor drug delivery. Trends Cancer. 2017;3(9):621630. doi:10.1016/j.trecan.2017.07.006

108. Cui X-J, He Q, Zhang J-M, Fan H-J, Wen Z-F, Qin Y-R. High-dose aspirin consumption contributes to decreased risk for pancreatic cancer in a systematic review and meta-analysis. Pancreas. 2014;43(1):135140. doi:10.1097/MPA.0b013e3182a8d41f

109. Molina MA, Sitja-Arnau M, Lemoine MG, Frazier ML, Sinicrope FA. Increased cyclooxygenase- 2 expression in human pancreatic carcinomas and cell lines: growth inhibition by nonsteroidal antiinflammatory drugs. Cancer Res. 1999;59(17):4356-4362.

110. Okami J, Yamamoto H, Fujiwara Y, et al. Overexpression of cyclooxygenase-2 in carcinoma of the pancreas. Clin Cancer Res. 1999;5(8):2018-2024.

111. Juuti A, Louhimo J, Nordling S, Ristimäki A, Haglund C. Cyclooxygenase-2 expression correlates with poor prognosis in pancreatic cancer. $J$ Clin Pathol. 2006;59(4):382-386. doi:10.1136/jcp.2005.026831

112. Matsubayashi H, Infante JR, Winter JM, et al. Tumor COX-2 expression and prognosis of patients with resectable pancreatic cancer. Cancer Biol Ther. 2007;6(10):1569-1575. doi:10.4161/ cbt.6.10.4711

113. Čeponytė U, Paškevičiūtė M, Petrikaitė V. Comparison of NSAIDs activity in COX-2 expressing and non-expressing 2D and 3D pancreatic cancer cell cultures. Cancer Manag Res. 2018;10:1543. doi:10.2147/CMAR.S163747

114. Lyons TR, O'brien J, Borges VF, et al. Postpartum mammary gland involution drives progression of ductal carcinoma in situ through collagen and COX-2. Nat Med. 2011;17(9):1109-1115. doi: $10.1038 / \mathrm{nm} .2416$

115. Itakura J, Ishiwata T, Friess $\mathrm{H}$, et al. Enhanced expression of vascular endothelial growth factor in human pancreatic cancer correlates with local disease progression. Clin Cancer Res. 1997;3(8):1309-1316.

116. Li S, Xu H-X, Wu C-T, et al. Angiogenesis in pancreatic cancer: current research status and clinical implications. Angiogenesis. 2019;22(1):15-36. doi:10.1007/s10456-018-9645-2

117. Pàez-Ribes M, Allen E, Hudock J, et al. Antiangiogenic therapy elicits malignant progression of tumors to increased local invasion and distant metastasis. Cancer Cell. 2009;15(3):220-231. doi:10.1016/j.ccr.2009.01.027

118. Ribatti D. Antiangiogenic therapy accelerates tumor metastasis. Leukemia Research. 2011;35(1):24-26. doi:10.1016/j. leukres.2010.07.038

119. Yin T, He S, Ye T, Shen G, Wan Y, Wang Y. Antiangiogenic therapy using sunitinib combined with rapamycin retards tumor growth but promotes metastasis. Transl Oncol. 2014;7(2):221229. doi:10.1016/j.tranon.2014.02.007

120. Terry S, Savagner P, Ortiz-Cuaran S, et al. New insights into the role of EMT in tumor immune escape. Mol Oncol. 2017;11 (7):824-846. doi:10.1002/1878-0261.12093

121. Bergers G, Hanahan D. Modes of resistance to anti-angiogenic therapy. Nat Rev Cancer. 2008;8(8):592-603. doi:10.1038/ nrc 2442

122. Van Cutsem E, Vervenne WL, Bennouna J, et al. Phase III trial of bevacizumab in combination with gemcitabine and erlotinib in patients with metastatic pancreatic cancer. J Clin Oncol. 2009;27 (13):2231-2237. doi:10.1200/JCO.2008.20.0238
123. Sahai V, Saif MW, Kalyan A, et al. A Phase I/II open-label multicenter single-arm study of FABLOx (Metronomic 5Fluorouracil Plus nab-Paclitaxel, Bevacizumab, Leucovorin, and Oxaliplatin) in patients with metastatic pancreatic cancer. $J$ Pancreatic Cancer. 2019;5(1):35-42. doi:10.1089/ pancan.2019.0012

124. Shen Y, Wang X, Lu J, et al. Reduction of liver metastasis stiffness improves response to bevacizumab in metastatic colorectal cancer. Cancer Cell. 2020;37(6):800-817. doi:10.1016/j. ccell.2020.05.005

125. Jacobetz MA, Chan DS, Neesse A, et al. Hyaluronan impairs vascular function and drug delivery in a mouse model of pancreatic cancer. Gut. 2013;62(1):112-120. doi:10.1136/gutjnl2012-302529

126. Provenzano PP, Cuevas C, Chang AE, Goel VK, Von Hoff DD, Hingorani SR. Enzymatic targeting of the stroma ablates physical barriers to treatment of pancreatic ductal adenocarcinoma. Cancer Cell. 2012;21(3):418-429. doi:10.1016/j.ccr.2012.01.007

127. Komar G, Kauhanen S, Liukko K, et al. Decreased blood flow with increased metabolic activity: a novel sign of pancreatic tumor aggressiveness. Clin Cancer Res. 2009;15(17):5511-5517. doi:10.1158/1078-0432.CCR-09-0414

128. Martin JD, Seano G, Jain RK. Normalizing function of tumor vessels: progress, opportunities, and challenges. Annu Rev Physiol. 2019;81:505-534. doi:10.1146/annurev-physiol-020518-114700

129. Stylianopoulos T, Munn LL, Jain RK. Reengineering the tumor vasculature: improving drug delivery and efficacy. Trends Cancer. 2018;4(4):258-259. doi:10.1016/j.trecan.2018.02.010

130. Nagathihalli NS, Castellanos JA, Shi C, et al. Signal transducer and activator of transcription 3 , mediated remodeling of the tumor microenvironment results in enhanced tumor drug delivery in a mouse model of pancreatic cancer. Gastroenterology. 2015;149 (7):1932-1943. doi:10.1053/j.gastro.2015.07.058

131. Kim SJ, Jung KH, Son MK, et al. Tumor vessel normalization by the PI3K inhibitor HS-173 enhances drug delivery. Cancer Lett. 2017;403:339-353. doi:10.1016/j.canlet.2017.06.035

132. Trindade A, Djokovic D, Gigante J, Mendonça L, Duarte A. Endothelial Dll4 overexpression reduces vascular response and inhibits tumor growth and metastasization in vivo. BMC Cancer. 2017;17(1):1-13. doi:10.1186/s12885-017-3171-2

133. Hamzah J, Jugold M, Kiessling F, et al. Vascular normalization in Rgs5-deficient tumours promotes immune destruction. Nature. 2008;453(7193):410-414. doi:10.1038/nature06868

134. Wei Y, Song S, Duan N, et al. MT1-MMP-Activated Liposomes to Improve Tumor Blood Perfusion and Drug Delivery for Enhanced Pancreatic Cancer Therapy. Adv Sci. 2020;7 (17):1902746. doi:10.1002/advs.201902746

135. Hosein AN, Brekken RA, Maitra A. Pancreatic cancer stroma: an update on therapeutic targeting strategies. Nat Rev Gastroenterol Hepatol. 2020;17(8):487-505. doi:10.1038/s41575-020-0300-1

136. Whatcott CJ, Diep CH, Jiang P, et al. Desmoplasia in primary tumors and metastatic lesions of pancreatic cancer. Clin Cancer Res. 2015;21(15):3561-3568. doi:10.1158/1078-0432.CCR-141051

137. Laklai H, Miroshnikova YA, Pickup MW, et al. Genotype tunes pancreatic ductal adenocarcinoma tissue tension to induce matricellular fibrosis and tumor progression. Nat Med. 2016;22 (5):497-505. doi:10.1038/nm.4082

138. Thompson CB, Shepard HM, O'Connor PM, et al. Enzymatic depletion of tumor hyaluronan induces antitumor responses in preclinical animal models. Mol Cancer Ther. 2010;9(11):30523064. doi:10.1158/1535-7163.MCT-10-0470

139. Hingorani SR, Harris WP, Beck JT, et al. Phase Ib study of PEGylated recombinant human hyaluronidase and gemcitabine in patients with advanced pancreatic cancer. Clin Cancer Res. 2016;22(12):2848-2854. doi:10.1158/1078-0432.CCR-15-2010 
140. Tempero MA, Van Cutsem E, Sigal D, et al. HALO 109-301: a randomized, double-blind, placebo-controlled, phase 3 study of pegvorhyaluronidase alfa (PEGPH20)+ nab-paclitaxel/gemcitabine (AG) in patients (pts) with previously untreated hyaluronan (HA)-high metastatic pancreatic ductal adenocarcinoma (mPDA). Am Soc Clin Oncol. 2020;1:587.

141. Bever KM, Sugar EA, Bigelow E, et al. The prognostic value of stroma in pancreatic cancer in patients receiving adjuvant therapy. Hpb. 2015;17(4):292-298. doi:10.1111/hpb.12334
142. Chen Y, Kim J, Yang S, et al. Type I collagen deletion in $\alpha$ SMA+ myofibroblasts augments immune suppression and accelerates progression of pancreatic cancer. Cancer Cell. 2021.

143. Zinger A, Koren L, Adir O, et al. Collagenase nanoparticles enhance the penetration of drugs into pancreatic tumors. ACS Nano. 2019;13(10):11008-11021. doi:10.1021/acsnano.9b02395

\section{Publish your work in this journal}

The International Journal of Nanomedicine is an international, peerreviewed journal focusing on the application of nanotechnology in diagnostics, therapeutics, and drug delivery systems throughout the biomedical field. This journal is indexed on PubMed Central, MedLine, CAS, SciSearch ${ }^{\mathbb{R}}$, Current Contents ${ }^{\mathbb{R}} /$ Clinical Medicine, $^{2}$
Journal Citation Reports/Science Edition, EMBase, Scopus and the Elsevier Bibliographic databases. The manuscript management system is completely online and includes a very quick and fair peer-review system, which is all easy to use. Visit http://www.dovepress.com/ testimonials.php to read real quotes from published authors. 